\title{
Pretilachlor poisoning: A rare case of a herbicide masquerading as organophosphate toxicity
}

\author{
Olita Shilpakar ${ }^{1}$, Bipin Karki ${ }^{2}$, and Bibek Rajbhandari ${ }^{3}$ \\ ${ }^{1}$ NAMS \\ ${ }^{2}$ Affiliation not available \\ ${ }^{3}$ Nepal Police Hospital
}

August 28, 2020

\begin{abstract}
Pretilachlor is a chloracetanilide herbicide whose acute intoxication in humans via ingestion has been rarely reported. We report a case of suicidal ingestion of the herbicide with similar clinical manifestations of organophosphate toxicity. Awareness among clinicians regarding such mimickers is the key to proper management of the patient.
\end{abstract}

\section{Pretilachlor poisoning: A rare case of a herbicide masquerading as organophosphate toxicity} Olita Shilpakar ${ }^{1}$, Bipin Karki ${ }^{2}$, Bibek Rajbhandari ${ }^{3}$

${ }^{1}$ Department of General Practice and Emergency Medicine, NAMS, Bir Hospital, Kathmandu, Nepal, ${ }^{2}$ Department of Critical Care Medicine, Om Hospital and Research Centre, Kathmandu, Nepal, ${ }^{3}$ Department of General Practice and Emergency Medicine, Nepal Police Hospital, Kathmandu, Nepal.

Correspondence: Dr. Olita Shilpakar, Department of General Practice and Emergency Medicine, NAMS, Bir Hospital, Kathmandu. E-mail: olitashilpakar1@gmail.com, Phone: +977-9841256959.

\section{ABSTRACT}

Pretilachlor is a chloracetanilide herbicide whose acute intoxication in humans via ingestion has been rarely reported. We report a case of suicidal ingestion of the herbicide with similar clinical manifestations of organophosphate toxicity. Awareness among clinicians regarding such mimickers is the key to proper management of the patient.

Keywords: bradycardia; poisoning; vomiting.

\section{KEY CLINICAL MESSAGE}

Acute oral intoxication of pretilachlor, a chloracetanilide herbicide, in humans can present with similar clinical manifestations of organophosphate toxicity. Clinicians should be aware of such mimickers for proper management of the patient.

\section{INTRODUCTION}

Chloracetanilides are a group of anilide herbicides which commonly include alachlor, butachlor, metachlor and pretilachlor. Pretilachlor is a broad spectrum systemic herbicide with the chemical name 2-chloro-2', 6'-diethyl-N-(2-propoxyethyl) acetanilide. It has excellent action against annual weeds, sedges and broadleaf weeds in rice fields. ${ }^{1}$ The mechanism of action of these group of drugs is still not clearly understood but is known to act by inhibiting the biosynthesis of fatty acids, lipids, proteins, flavonoids, etc. Chronic 
exposure to these group of herbicides has shown probable carcinogenic effects, however, acute toxicity from pretilachlor in humans has not been reported yet. ${ }^{2}$ Acute pretilachlor intoxication via ingestion can be mistaken for commonly encountered pesticides like organophosphates resulting in fallacious management of the patient. We report a case of a forty-two-years male who presented to the emergency room following suicidal ingestion of the herbicide mimicking clinical manifestations of organophosphate toxicity like vomiting, excessive lacrimation, bowel and bladder incontinence, bradycardia and hypotension and complete recovery following supportive management.

\section{CASE REPORT}

A forty-two-years male presented to the emergency room with the alleged history of suicidal ingestion of an unknown poison two hours back following a family dispute. The patient immediately had two episodes of non bilious non projectile vomiting followed by excessive lacrimation, bowel and bladder incontinence and dizziness within two hours of ingestion of the poison. There was no history of shortness of breath, chest pain, loss of consciousness or seizures. He did not have any past medical or psychiatric history or similar suicidal attempts in the past. On examination in the emergency room, the patient was drowsy but followed verbal commands. He had a radial pulse of 50 beats per minute, blood pressure of $90 / 50 \mathrm{~mm} \mathrm{Hg}$, respiratory rate of 16 breaths/minute, temperature of 98.6 degrees Fahrenheit and oxygen saturation of $94 \%$ in room air. His pupils were 3 millimeters in size bilaterally and reacting to light. Furthermore, the peculiar garlicky smell of organophosphate was not detected from his breath. His chest auscultation was clear, abdomen was soft and non tender and the remaining systemic examination did not reveal any abnormality.

With the provisional diagnosis of a possible organophosphorus poisoning, the patient was managed initially by administering an intravenous dose of atropine $0.6 \mathrm{mg}$ following which his heart rate increased to 100 beats per minute showing a normal sinus rhythm in the 12 lead electrocardiogram. Dermal decontamination was done by removing the patient's vomitus soaked clothing. The skin was cleaned thoroughly with soap water and gastric lavage was performed simultaneously. A bolus dose of $500 \mathrm{ml}$ of intravenous $0.9 \%$ Normal Saline and an intravenous dose of pralidoxime 2 grams over 30 minutes were administered. His investigations comprising a full blood count, arterial blood gas, renal function tests, a random blood sugar level, liver function tests, a coagulation profile and a serum cholinesterase level were all within normal limits. A chest radiograph and an ultrasonogram of the abdomen and pelvis did not reveal any abnormalities.

A bottle of the herbicide pretilachlor $50 \%$ emulsifiable concentrate (EC) was retrieved from his room and was brought to the emergency room by his son after two hours. The patient confirmed to have consumed the full bottle containing $250 \mathrm{ml}$ of the herbicide. Close monitoring with continuous supportive management was done for 24 hours with intravenous fluids and antiemetics following which he gradually became asymptomatic. His heart rate remained within the normal range, his blood pressure normalized and a normal urine output was maintained throughout the hospital stay. Symptoms of lacrimation and incontinence subsided. Repeat blood counts, renal and liver functions were within normal ranges. He was kept under observation with strict hemodynamic monitoring for the next two days which was uneventful. He was discharged from the hospital on the third day after a behavioural counselling session. He was in good health during a one-week follow up in the outpatient department.

\section{DISCUSSION}

Pretilachlor is a synthetic chloracetanilide herbicide used effectively in annual grasses and broad-leaved weeds including Echinochloa Beauvois, Cyperus difformis and sedges in rice and paddy fields. Pretilachlor is generally marketed in a $50 \%$ emulsifiable concentrate formulation. It appears as a colourless and odourless liquid. It contains an ethoxylated vegetable oil as the emulsifying agent and alkyl aryl sulphonate of calcium salt as the surfactant. ${ }^{1}$

Chronic exposure to chloracetanilide in vitro and vivo studies have shown that it has a role in causing neurotoxicity, genotoxicity and carcinogenicity. ${ }^{2,3}$ Acute oral exposure might have a dissimilar effect on humans but such cases have been rarely reported to date. A retrospective study of 35 patients with acute oral chloracetanilide poisoning concluded that although it was found to be of low toxicity in most of the 
patients, three patients were comatose and one patient died 24 hours after the exposure. ${ }^{4}$ Another study by Lo et al in 113 patients with oral exposure to chloracetanilides like alachlor and butachlor suggested that around one fourth of the patients were asymptomatic, the rest had vomiting and neurological symptoms ranging from drowsiness to central nervous system depression and three fatalities after manifesting profound hypotension and coma. ${ }^{5}$

Misdiagnosis of pretilachlor poisoning as organophosphorus toxicity in the emergency room could be a major drawback and may lead to faulty management. In our case, the patient displayed several similar clinical features that are encountered with organophosphates like vomiting, excessive lacrimation, bowel bladder incontinence, bradycardia and hypotension. These hypersecretory effects like salivation, lacrimation, urination, defecation, gastrointestinal motility, emesis and miosis in case of organophosphate poisoning are due to the overstimulation of muscarinic acetylcholine receptors in the parasympathetic system. Acute muscarinic effects on the heart in the form of bradycardia and hypotension can be life threatening. ${ }^{6}$ However, there was no miosis or the presence of the characteristic garlicky odour of organophosphorus toxicity which hinted towards an alternative diagnosis. His serum cholinesterase level was also within normal limit which in case of organophosphates are seen to decrease. ${ }^{6}$

No antidote is yet known to be available for pretilachlor poisoning so the mainstay of treatment is symptomatic with initial stabilization of the patient, decontamination, intravenous fluid resuscitation and close hemodynamic monitoring. ${ }^{7}$ In our case, symptomatic bradycardia was alleviated with atropine sulphate administration and the patient became normotensive following intravenous fluids. Therefore, further studies are recommended which would elaborate more on the mechanism of action of this drug on the human body and the definitive management to combat its toxic effects. ${ }^{8}$

\section{CONCLUSION}

This case highlights that despite exhibiting similar clinical manifestations like that of organophosphorus poisoning, initial stabilization, close monitoring, and supportive treatment are the three important aspects for a speedy recovery in pretilachlor poisoning. Education and awareness among the treating physicians regarding mimickers like organophosphates is an important point to be taken into consideration from this case report. The importance of retrieving the poison container wherever possible for the identification of the active compound is emphasized. Redesigning of containers with precautionary warning labels for the general public and restricted availability of this herbicide over the counter are highly recommended.

\section{ACKNOWLEDGEMENTS}

The authors are grateful to the patient and his family for providing support regarding the preparation of the report.

\section{CONFLICT OF INTEREST:}

None declared.

\section{AUTHOR CONTRIBUTION:}

OS: was involved in the patient care and management and designed the study. OS and BK: prepared the initial draft of the manuscript. OS, BK and BR: edited the draft and reviewed the manuscript. All authors approved the final version of the manuscript and agreed to be accountable for any aspects related to the accuracy or integrity of the work.

ORCID:

Olita Shilpakar https://orcid.org/0000-0002-4437-531X

\section{REFERENCES}

1. Lewis, K.A., Tzilivakis, J., Warner, D. and Green, A. An international database for pesticide risk assessments and management. Human and Ecological Risk Assessment: An International Journal.2016; 22(4), 
1050-1064. doi: 10.1080/10807039.2015.1133242

2. Dearfield KL, McCarroll NE, Protzel A, Stack HF, Jackson MA, WatersMD. A survey of EPA/OPP and open literature on selected pesticide chemicals.II. Mutagenicity and carcinogenicity of selected chloroacetanilides and related compounds. Mutat Res 1999; 443: 183-221. PMID: 10415440 doi: 10.1016/s13835742(99)00019-8

3. El-Baz MAH, El-Deek SEM, Nsar AY, El-Maali EA, Abdel Hafez FF, Amin AF. Prenatal Pesticide Exposure: Meconium as a Biomarker and Impact on Fetal Weight. J Environ Anal Toxicol 2015;5: 268. doi:10.4172/2161-0525.1000268

4. Seok SJ, Choi SC, Gil HW, Yang JO, Lee EY, Song HY, Hong SY. Acute Oral Poisoning Due to Chloracetanilide Herbicides. J Korean Med Sci. 2012 Feb;27(2):111-114. https://doi.org/10.3346/jkms.2012.27.2.111

5. Lo YC, Yang CC, Deng JF. Acute alachlor and butachlor herbicide poisoning. Clin Toxicol (Phila) 2008; 46: 716-21. PMID: 19238733 doi: 10.1080/15563650701704834

6. Eddleston M, Buckley NA, Eyer P, Dawson AH. Management of acute organophosphorus pesticide poisoning. Lancet.2008;371(9612):597-607. doi:10.1016/S0140-6736(07)61202-1

7. Rakesh Soni,Sushant Kumar Verma. Acute toxicity and behavioural responses in Clariasbatrachus (Linnaeus) exposed to herbicide pretilachlor. Heliyon 4 (2018) e01090.doi: 10.1016/j.heliyon.2018.e01090

8. Dawson AH, Eddleston M, Senarathna L, Mohamed F, Gawarammana I, et al. Acute Human Lethal Toxicity of Agricultural Pesticides: A Prospective Cohort Study. PLoS Med 2010;7(10): e1000357. doi:10.1371/journal.pmed.1000357 